\title{
Scheduling Open-Nested Transactions in Distributed Transactional Memory
}

\author{
Junwhan Kim, Roberto Palmieri, and Binoy Ravindran \\ ECE Department, Virginia Tech, Blacksburg, VA, 24061 \\ \{junwhan, robertop, binoy\}@vt.edu
}

\begin{abstract}
Distributed transactional memory (DTM) is a powerful concurrency control model for distributed systems sparing the programmer from the complexity of manual implementation of lock-based distributed synchronization. We consider Herlihy and Sun's dataflow DTM model, where objects are migrated to invoking transactions, and the open nesting model of managing inner (distributed) transactions. In this paper we present DATS, a dependency-aware transactional scheduler, that is able to boost the throughput of open-nested transactions reducing the overhead of running expensive compensating actions and abstract locks in the case of outer transaction aborts. The contribution of the paper is twofold: (A) DATS allows the commutable outer transactions to be validated concurrently and (B) allows the non-commutable outer transactions, depending on their inner transactions, to commit be committed before others without dependencies.
\end{abstract}

\section{Introduction}

Transactional Memory (TM) is an emerging innovative programming paradigm for transactional systems. The main benefit of TM is synchronization transparency in concurrent applications. In fact, leveraging the proven concept of atomic and isolated transactions, TM spares programmers from the pitfalls of conventional manual lock-based synchronization, significantly simplifying the development of parallel and concurrent applications. Moreover lock-based concurrency control suffers from programmability, scalability, and composability challenges [13] and TM promises to alleviate these difficulties. In TM, the developer simply organizes read and write operations on shared objects as transactions and leaves the responsibility of executing those transactions to the TM, ensuring atomicity, consistency and isolation. Two transactions conflict if they access to the same object and at least one access is a write. The contention manager, the component in TM responsible for resolving conflicts among concurrent transactions, typically aborts one and allows the other to commit, yielding (the illusion of) atomicity. Aborted transactions are typically re-started after rollingback the changes in memory.

The Transaction Scheduler (TS) is the component that supports the contention manager in making a decision on how to resolve conflicts (which transaction to abort). The goal of the TS is to order concurrent transactions as to avoid or minimize conflicts (and thereby aborts). 
The hazards of manual implementation of lock-based concurrency control increases in distributed settings due to an additional synchronization level among nodes in the system. Distributed STM (DTM) has been motivated as an alternative to distributed lock-based concurrency control. DTM can be classified based on the system architecture: cache-coherent DTM (cc DTM) [1423, in which a set of nodes communicate by message-passing links over a communication network, and a cluster model (cluster DTM) [621, in which a group of linked computers work closely together to form a single computer. cc DTM uses a cache-coherence protocol [8,14] to locate and move objects in the network.

Support for nesting (distributed) transactions is essential for DTM, for the same reasons that they are so for multiprocessor TM - i.e., code composability, performance, and fault-management [20 24 25]. Three types of nesting have been studied for multiprocessor TM: flat, closed, and open. If an inner transaction $I$ is flat-nested inside its outer transaction $A, A$ executes as if the code for $I$ is inlined inside $A$. Thus, if $I$ aborts, it causes $A$ to abort. If $I$ is closed-nested inside $A$ [19], the operations of $I$ only become part of $A$ when $I$ commits. Thus, an abort of $I$ does not abort $A$, but $I$ aborts when $A$ aborts. Finally, if $I$ is open-nested inside $A$, then the operations of $I$ are not considered as part of $A$. Thus, an abort of $I$ does not abort $A$, and vice versa.

The differences between the nesting models are shown in Figure 1 in which there are two transactions containing a nested-transaction. With flat nesting, transaction $T_{2}$ cannot execute until transaction $T_{1}$ commits. $T_{2}$ incurs full aborts, and thus has to restart from the beginning. Under closed nesting, only $T_{2}$ 's inner-transaction needs to abort and be restarted while $T_{1}$ is still executing. The portion of work $T_{2}$ executes before the data-structure access does not need to be retried, and $T_{2}$ can thus finish earlier. Under open nesting, $T_{1}$ 's inner-transaction commits independently of its outer, releasing memory isolation over the shared data-structure. $T_{2}$ 's inner-transaction can therefore proceed immediately, thus enabling $T_{2}$ to commit earlier than in both closed and flat nesting.

The flat and closed nested models have a clear negative impact on large monolithic transactions in terms of concurrency. In fact, when a large transaction is aborted all its flat/closed-nested transactions are also aborted and rolled-back, even if they do not conflict with any other transaction. Closed nesting potentially offers better performance than flat nesting because the aborts of closed-nested inner transactions do not affect their outer transactions. However the opennesting approach outperforms both in terms of concurrency allowed. When an open-nested transaction commits, its modifications on objects become immediately visible to other transactions, allowing those transactions to start using those objects without a conflict, increasing concurrency 20. In contrast, if the inner transactions are closed- or flat-nested, then those object changes are not made visible until the outer transaction commits, potentially causing conflicts with other transactions that may want to use those objects.

To achieve high concurrency in open nesting, inner transactions have to implement abstract serializability [27. If concurrent executions of transactions result in the consistency of shared objects at an "abstract level", then the executions are said to be abstractly serializable. If an inner transaction $I$ commits, $I$ 's 
modifications are immediately committed in memory and $I$ 's read and write sets are discarded. At this time, $I$ 's outer transaction $A$ does not have any conflict with $I$ due to memory accessed by $I$. Thus, programmers consider the internal memory operations of $I$ to be at a "lower level" than $A$. $A$ does not consider the memory accessed by $I$ when it checks for conflicts, but $I$ must acquire an abstract lock and propagates this lock for $A$. When two operations try to acquire the same abstract lock, the open nesting concurrency control is responsible for managing this conflict (so this is defined "abstract level").

If an outer transaction (with opennested inner transactions) aborts, all of its (now committed) open-nested inner transactions must rollback and their actions must be undone to ensure transaction serializability. Thus, with the open nesting model, programmers must provide a compensating action for each open-nested transaction [2]. In scenarios in which outer transactions increasingly encounter conflicts after that a large number of their open-nested transactions have committed (e.g., long running transactions), the overall performance could collapse and all the benefits of the open-nesting approach vanish due to the processing of compensating actions to undo the modifications provided by the committed open-nested

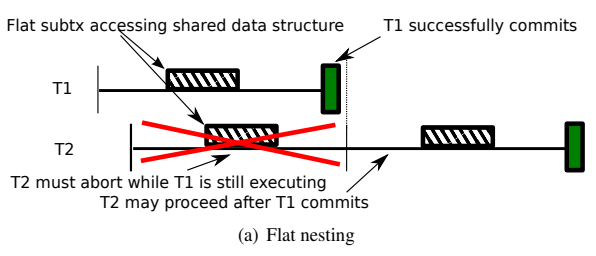

Closed subtx accessing shared data structure T1 successfully commits

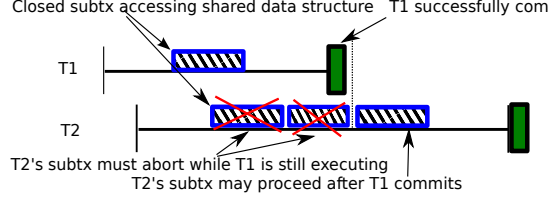

(b) Closed nesting

Open subtx accessing shared data structure

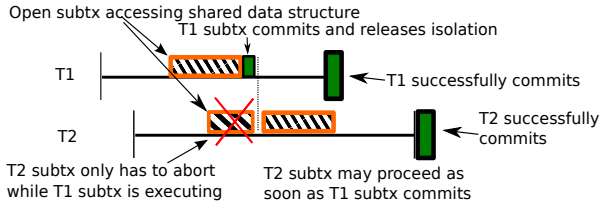

(c) Open nesting

Fig. 1. Two transactions under flat, closed and open nesting (From [24]) transactions. In closed nesting, since closed-nested transactions are not committed in memory until the outer transaction commits (nested transactions' changes are visible only to the outer), no undo (e.g., compensation) is required. Moreover, in scenarios in which the load of the system is high, the probability of having concurrent conflicting transactions grows and with it the probability to abort outer transactions with a number of open-nested transactions (already committed in memory). Aborting outer transactions with dependencies, instead of alleviating the load of the system, will increase it due to the execution of compensating actions, possibly bringing the system toward dangerous states.

We focus on these problems: the overhead of compensating actions and abstract locks in the open-nested model. Our goal is to boost performance of nested transactions in DTM by increasing concurrency and reducing the aforementioned overhead through transactional scheduling. For these reasons, we designed a scheduler, called the Dependency-Aware Transactional Scheduler (or DATS). DATS is responsible for helping the concurrency control minimizing the number of outer-transactions aborted. In order to do that, DATS relies on the notions of commutable transactions and transaction dependencies. 
Commutable Transactions. Two transactions are defined as commutable if they conflict and they leave the state of the shared data-set consistent even if validated and committed concurrently. A very intuitive example of commutativity is when two operations, $\operatorname{call1}(X)$ and $\operatorname{call2}(X)$, both access the same object $X$ but different fields of $X$ (See Section 3.2 for discussion about commutativity).

Transaction Dependencies. An outer transaction materializes dependencies with its inner transactions if (i) the inner transactions accesses the outer writeset for performing local computation or (ii) the results of outer processing are used to decide whether or not to invoke an inner transaction.

DATS is able to detect commutable transactions and validate/commit them, avoiding useless aborts. In the case of non-commutable transactions, DATS identifies how much each outer transaction depends on its inner transactions and schedules the outer transaction with the highest dependency to commit before other outer transactions with lower or no dependencies. Committing this outer transaction prevents its dependent inner transactions from aborting and reduces the number of compensating actions. Moreover, even though the other outer transactions abort, their independent inner transactions will be preserved, resulting in a reduced number of compensating actions and abstract locks without violating the correctness of the object.

We implemented DATS in a Java DTM framework, called HyFlow [22, and conducted an extensive experimental study involving both micro-benchmarks (e.g., Hash Table, Skip-, Linked-List) and a real application benchmark (TPCC). Our study reveals that throughput is improved by up to $1.7 \times$ in microbenchmarks and up to $2.2 \times$ in TPC-C over open-nested DTM without DATS. To the best of our knowledge, DATS is the first ever scheduler that boosts throughput with open-nested transactions in DTM.

The rest of the paper is organized as follows. We present preliminaries of the DTM model and state our assumptions in Section 2. We describe DATS and analyze its properties in Section 3 Section 4 reports our evaluation. We overview past and related efforts in Section 5 , and Section 6 concludes the paper.

\section{Preliminaries and System Model}

We consider a distributed system which consists of a set of nodes $N=\left\{n_{1}, n_{2}, \cdots\right\}$ that communicate with each other by message-passing links over a network. Similar to [14, we assume that the nodes are scattered in a metric space.

Transaction Model. A set of shared objects $O=\left\{o_{1}, o_{2}, \ldots\right\}$ are distributed in the network among nodes. A transaction is defined as a sequence of requests, each of which is a read or write operation request to an a single object in $O$. An execution of a transaction is a sequence of timed operations that ends by either a commit (success) or an abort (failure). A transaction is in three possible states: live, aborted, or committed. Each transaction has a unique identifier and is invoked by a node in the system. We consider the data flow DTM model [14]. In this model, transactions are immobile and objects move from node to node to invoking transactions. Each node has a TM proxy that provides interfaces 
allowing the local application to interact with the other proxies located on other nodes. When a transaction $T_{i}$ at node $n_{i}$ requests object $o_{j}$, the TM proxy of $n_{i}$ first checks whether $o_{j}$ is in its local cache. If the object is not present, the proxy invokes a distributed cache-coherence protocol (CC) to fetch $o_{j}$ in the network.

Atomicity, Consistency, and Isolation. We use the Transactional Forwarding Algorithm with Open Nesting (TFA-ON) [24, which extends the TFA algorithm 22] (which originally does not provide any transaction nesting support), to manage flat, closed and open-nested transactions. TFA provides early validation of remote objects, guarantees a consistent view of shared objects between distributed transactions, and ensures atomicity for object operations in presence of asynchronous clocks. The early validation of remote objects means that a transaction validated first commits its objects successfully. Validation in distributed systems includes global registration of object ownership. TFA is responsible for caching local copies of remote objects and changing the ownership.

TFA-ON changes the scope of object validations. The behavior of open-nested transactions under TFA-ON is similar to the behavior of regular transactions under TFA. In addition, TFA-ON manages the abstract locks and the execution of commit and compensating actions 24]. To provide conflict detection at the abstract level, an abstract locking mechanism has been integrated into TFA-ON. Abstract locks are acquired only at commit time, once the inner transaction is verified to be conflict free at the low level. The commit protocol requests the abstract lock of an object from the object owner and the lock is released when its outer transaction commits. To abort an outer transaction properly, a programmer provides an abstract compensating action for each of its inner transaction to revert the data-structure to its original semantic state.

TFA-ON is the first ever implementation of a DTM system with support for open-nested transactions 24]. DATS has been integrated in TFA-ON.

\section{The DATS Scheduler}

\subsection{Motivations}

Figure 2 shows an example of open-nested transactions with compensating actions and abstract locks. Listings 1.1 and 1.2 in Figure 2 illustrate two outer transactions, $T_{1}$ and $T_{2}$, and an inner transaction in Listing 1.3. The inner transaction INSERT includes an insert operation in a Linked List. $T_{1}$ has a delete operation with a value. If the operation of $T_{1}$ executes successfully, its inner transaction INSERT executes. Conversely, regardless of the success of $T_{2}$ 's delete operation, its inner transaction INSERT will execute. OnCommit and OnAbort, which include a compensating action, are registered when the inner transaction commits. If the outer transaction (i.e., $T_{1}$ or $T_{2}$ ) commits, OnCommit executes. When the inner transaction commits, its modification becomes immediately visible for other transactions. Thus, if the inner transaction commits, and its outer transaction $T_{1}$ or $T_{2}$ aborts, a delete operation as a compensating action (described in OnAbort) executes. Let us assume that $T_{2}$ aborts, and OnAbort executes. Even though $T_{2}$ 's inner transaction (INSERT) does not depend on its 
Listing 1.1. Transaction $T_{1}$

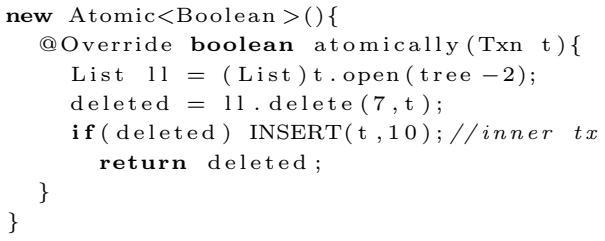

Listing 1.2. Transaction $T_{2}$

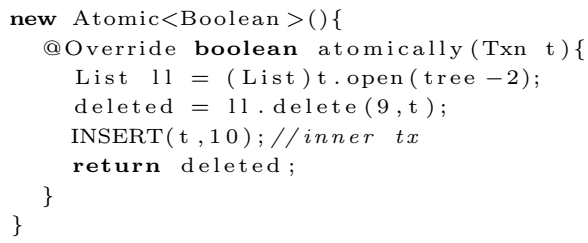

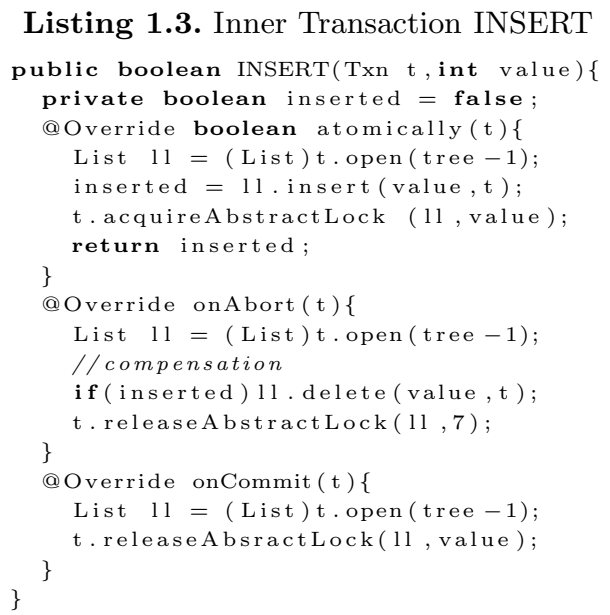

Fig. 2. Two open-nested transactions with abstract locks and compensating actions

delete operation, unlike $T_{1}$, OnAbort will execute. Thus, the conflict of object "tree- 2 " in $T_{2}$ causes the execution of compensating action on object "tree- 1 " in INSERT. The INSERT operation acquires the abstract lock again when it restarts. Finally, whenever an outer transaction aborts, its inner transaction must execute a compensating action, regardless of the operation's dependencies.

This drawback is particularly evident in distributed settings. In fact, distributed transactions typically have an execution time several orders of magnitude bigger than in a centralized STM, due to communication delays that are incurred in requesting and acquiring objects [16]. If an outer transaction aborts, clearly the impact of the time needed for running compensating actions and for acquiring abstract locks for distributed open-nested transactions is exacerbated due to the communication overhead. Moreover it increases the likelihood of conflicts, drastically reducing concurrency and degrading performance.

Motivated by these observations, we propose the DATS scheduler for opennested DTM. DATS, for each outer transaction $T_{a}$, identifies the number of inner transactions depending from $T_{a}$ and schedules the outer transactions with the greatest number of dependencies to validate first and (hopefully) commit. This behavior permits the transactions with high compensation overhead to commit; the remaining few outer transactions that are invalidated will be restarted excluding their independent inner transactions to avoid useless compensating actions and acquisition of abstract locks. In the next subsection the meaning of dependent transactions for DATS will be described.

\subsection{Abstract and Object Level Dependencies}

We consider two types of dependencies among transactions.

Abstract Level Dependency. The first is called abstract level dependency (ALD) and it indicates the dependency between an outer transaction and its inner 


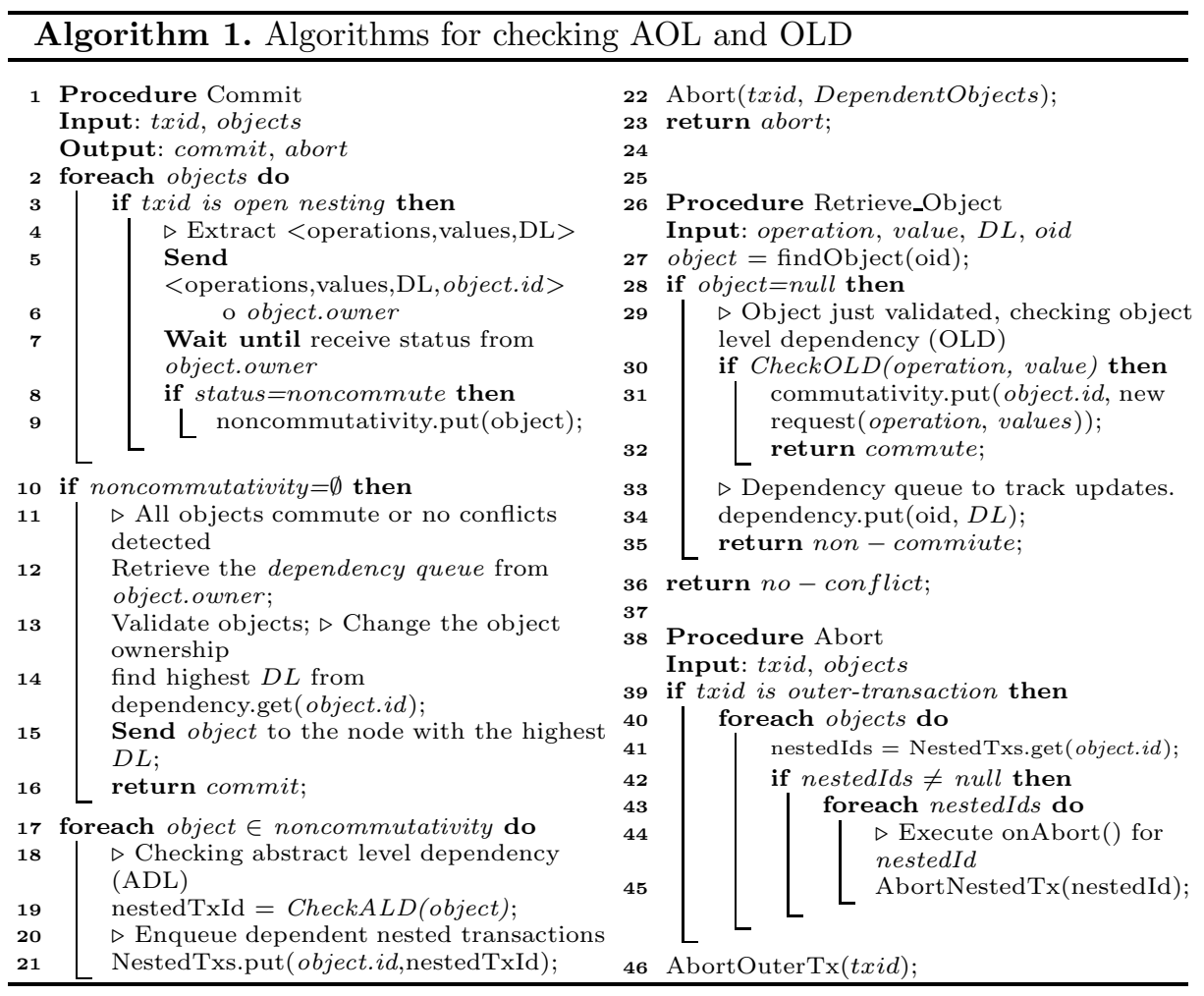

transactions at an abstract level. We define the dependency level $(D L)$ as the number of inner transactions that will execute OnAbort when the outer transactions abort. For example, $T_{1}$ illustrated in Figure 2 depends on its INSERT due to the deleted variable. Thus, DATS detects a dependency between $T_{1}$ and its INSERT (its inner transaction) because the delete operations in $T_{1}$ shares the variable deleted with the conditional if statement declared for executing INSERT. In this case, the $D L=1$ for $T_{1}$. Conversely, $T_{2}$ executes INSERT without checking any pre-condition so its $D L=0$ because $T_{2}$ does not have dependencies with its inner transactions. The purpose of the abstract level dependency is to avoid unnecessary compensating actions and abstract locks. Even though $T_{2}$ aborts, OnAbort in INSERT will not be executed because its $D L=0$, and the compensating action will not be processed. Meanwhile, executing OnAbort implies running INSERT and acquiring the abstract lock again when $T_{2}$ restarts.

Summarizing, aborting outer transactions with smaller $D L$ s leads to a reduced number of compensating actions and abstract lock acquisitions. Such identification can be done automatically at run-time by DATS using byte-code analysis or relying on explicit indication by the programmer. The first scenario is completely transparent from the application point of view but in some cases could add additional overhead. The second approach, although it requires the collaboration 
of the developer, is more flexible because it allows the programmer to bias the behavior of the scheduler. In fact, even though the logic of an outer transaction reveals a certain number of dependencies, the programmer may want to force running compensations in case of an abort. This can be done by simply changing the value of $D L$ associated to the outer transaction.

Object Level Dependency. The second is called object level dependency (OLD) and it indicates the dependency among two or more concurrent transactions accessing the same shared object. For example, in Figure 2, $T_{1}$ depends on $T_{2}$ because they share the same object "tree-2". If $T_{1}$ and $T_{2}$ work concurrently, a conflict between them occurs. However, delete $(7)$ of $T_{1}$ and delete $(9)$ of $T_{2}$ commute because they are two operations executing on the same object ("tree-2") but accessing different items (or fields when applicable) of the object (item "7" and item "9"). We recall that, two operations commute if applying them in either order they leave the object in the same state and return the same responses [12. DATS detects object level dependency at transaction commit phase, splitting the validation phase into two. Say $T_{a}$ is the transaction that is validating. In the first phase, $T_{a}$ checks the consistency of the objects requested during the execution. If a concurrent transaction $T_{b}$ has requested and already committed a new version of some object requested by $T_{a}$, then $T_{a}$ aborts in order to avoid isolation corruption. After the successful completion of the first phase of $T_{a}$ 's validation, DATS detects the object level dependencies among concurrent transactions that are validating with $T_{a}$ in the second phase. To do that, DATS relies on the notion of commutativity already introduced at the end of Section 1. Suppose $T_{a}$ and $T_{b}$ are conflicting transactions but simultaneously validating. If all of $T_{a}$ 's operations commute with all of $T_{b}$ 's operations, they can proceed to commit together avoiding a useless abort. Otherwise one of $T_{a}$ or $T_{b}$ must be aborted. This scheduler is in charge of the decision (see next sub-section).

In order to compute commutativity, DATS joins two supports. In the first, the programmer annotates each transaction class with the fields accessed. The second is a field-based timestamping mechanism, used for checking the fieldlevel invalidation. The goal is to reduce the granularity of the timestamp from object to field. With a single object timestamp, it is impossible to detect commutativity because of fields modifications. In fact, writes to different fields of the same object are all reflected with the increment of the same object timestamp. In order to do that efficiently, DATS exploits the annotations provided by the developer on the fields accessed by the transaction to directly point only to the interested fields (instead of iterating on all the object fields, looking for the ones modified). On such fields, it uses field-based timestamping to detect object invalidation.

The purpose of the object level dependency is to enhance concurrency of outer transactions. Even though inner transactions terminate successfully, aborting their outer transactions affects these inner transactions (due to compensation). Thus, DATS checks for the commutativity of conflicting transactions and permits them to be validated, reducing the aborts. 


\subsection{Scheduler Design}

We designed DATS using abstract level dependencies and object level dependencies. In Figure 1 is presented the pseudo-code with the procedures used by DATS for detecting ALD and OLD at validation/commit time. When outer transactions are invoked, the $D L$ with their inner transactions is checked. When the outer transactions request an object from its owner, the requests with their $D L$ s will be sent to the owner and moved into its scheduling queue. The object owner maintains the scheduling queue holding all the ongoing transactions that have requested the object with their $D L$ s. When $T_{1}$ (one of the outer transactions) validates an object, we consider two possible scenarios. First, if another transaction $T_{2}$ tries to validate the same object, a conflict between $T_{1}$ and $T_{2}$ is detected on the object. Thus, DATS checks for the object level dependency. If $T_{1}$ and $T_{2}$ are independent (according to the object level dependency rules), DATS allows $T_{1}$ and $T_{2}$ to proceed with the validation. Otherwise, the transaction with lower $D L$ will be aborted. In this way, dependent transactions with the minimal cost of abort and compensating actions are aborted and restarted, permitting transactions with a costly abort operation to commit.

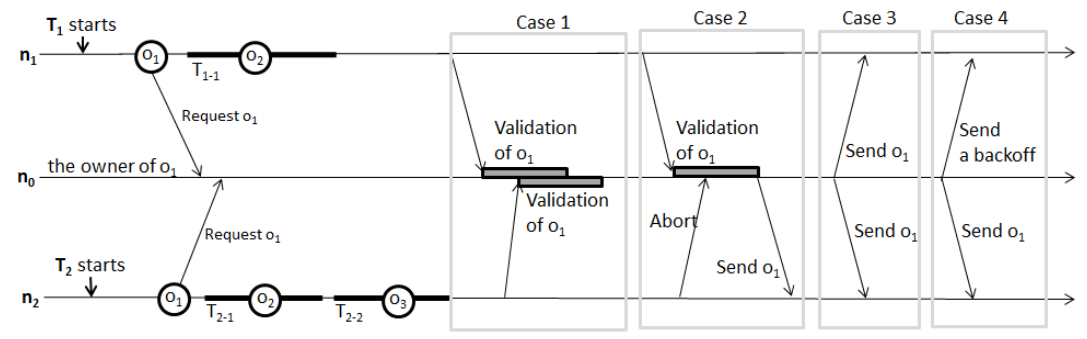

Fig. 3. Four Different Cases for Two Transactions $T_{1}$ and $T_{2}$ in DATS

Figure 3 illustrates an example of DATS with two transactions $T_{1}$ and $T_{2}$ invoked on nodes $n_{1}$ and $n_{2}$, respectively. The transaction $T_{1}$ has a single innertransaction and $T_{2}$ has two nested transactions. Let us assume that $T_{1}$ 's $D L=1$ and $T_{2}$ 's $D L=2$. The circles indicate written objects. The horizontal line corresponds to the status of each transaction described in the time domain. Figure 3 shows four different cases when $T_{1}$ and $T_{2}$ terminate. When $T_{1}$ and $T_{2}$ are invoked, DATS analyzes their $D L \mathrm{~s}$, operations, and values. When $T_{1}$ requests $o_{1}$ from $n_{0}$, the meta-data for $D L \mathrm{~s}$, operations and values of $o_{1}$ will be sent to $n_{0}$. These are moved to the scheduling queue of $n_{0}$. We consider four different cases regarding the termination of $T_{1}$ and $T_{2}$.

Case 1. $T_{1}$ and $T_{2}$ validate concurrently $o_{1}$. DATS checks for the object level dependency. If $T_{1}$ and $T_{2}$ are not dependent at the object level (i.e., the operations of $T_{1}$ and $T_{2}$ over $o_{1}$ commute), $T_{1}$ and $T_{2}$ commit concurrently.

Case 2. $T_{1}$ starts to validate and detects it is dependent with $T_{2}$ (that is still executing) at the object level on the object $o_{1}$. In this case $T_{2}$ will abort due to early validation. When $T_{1}$ commits, the updated $o_{1}$ is sent to $n_{2}$. 
Case 3. Another transaction committed $o_{1}$ before $T_{1}$ and $T_{2}$ validate. If $T_{1}$ and $T_{2}$ are not dependent at the object level, $o_{1}$ is sent to $n_{1}$ and $n_{2}$ simultaneously as soon as the transaction commits.

Case 4. Another transaction committed $o_{1}$ before $T_{1}$ and $T_{2}$ validate. If $T_{1}$ and $T_{2}$ are dependent at the object level, DATS checks for the abstract level dependency, and $o_{1}$ is sent to $n_{2}$ because $T_{2}$ 's $D L$ is larger than that of $T_{1}$. Aborting $T_{1}$, the scheduler is forced to run a single compensation (for $T_{1-1}$ ) instead of two compensations $\left(T_{2-1}\right.$ and $\left.T_{2-2}\right)$ in case of $T_{2}$ 's abort. Further, considering the case in which the $D L$ of $T_{1}$ is 0 , the abort of $T_{1}$ does not affect $T_{1-1}$. In fact, its execution will be preserved and only the operations of $T_{1}$ will be re-executed.

\section{Implementation and Experimental Evaluation}

Experimental Setup. We implemented DATS in the HyFlow DTM framework [22 25]. We cannot compare our results with any competitor, as none of the DTMs that we are aware of support open nesting and scheduling. Thus, we compared DATS under TFA-ON (DATS) with only TFA-ON (OPEN) [24], closed nested transaction (CLOSED) [25, and flat nested transaction (FLAT). We contrast with CLOSED and FLAT to show that OPEN does not always perform better than them, while DATS consistently outperforms OPEN.

We assess the performance of DATS using Hash Table, Skip List and Linked List as micro-benchmarks, TPC-C [7] as a real-application benchmark. Our testbed is comprised of 10 nodes, each one is an Intel Xeon $1.9 \mathrm{GHz}$ processor with 8 CPU cores. We varied the number of application threads performing operations for each node from 1 to 8 , considering a spectrum between 2 and 80 concurrent threads in the system. We measured the throughput (number of committed transactions per second). All data-points reported are the result of multiple executions, so plots present for each data-point the mean value and the error-bar. In order to assess the goodness of DATS we also present the percentage of aborted transactions and the scheduler overhead.

Benchmarks. The Skip List and Linked List benchmarks are data structures maintaining sorted and unsorted, lists of items, respectively, whereas Hash Table is an associative array mapping keys to values. We configured the benchmarks with the small number of objects and a large number of inner transactions - eight inner transactions per transaction and ten objects, incurring high contention.

Regarding TPC-C, the write transactions consist of update, insert, and/or delete operations accessing a database of nine tables maintained in memory, where each row has a unique key. Multiple operations commute if they access a row (or object) with the same key and modify different columns. We configured the benchmark with a limited number of warehouses (\#3) in order to generate high conflicts. We recall that, in the data flow model, objects are not bound on fixed nodes but move, increasing likelihood of conflicts.

Evaluation. Figures 4, 5] and 6( $a-f)$ show the throughput of micro-benchmarks under $10 \%$ and $90 \%$ of read transactions. The purpose of DATS is to reduce the 


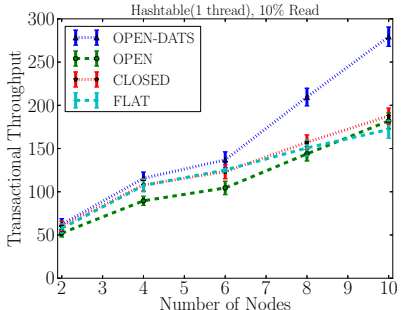

(a) HT (1 thread), 10\% Read

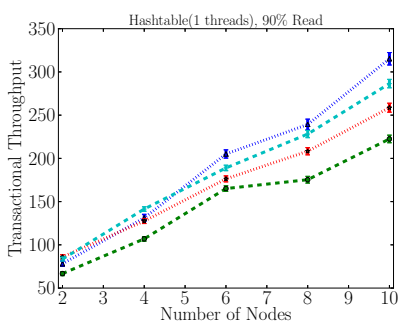

(d) HT (1 thread), 90\% Read

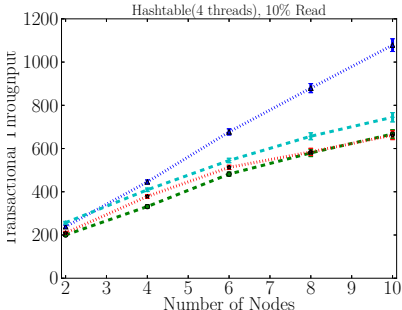

(b) HT (4 threads), 10\% Read

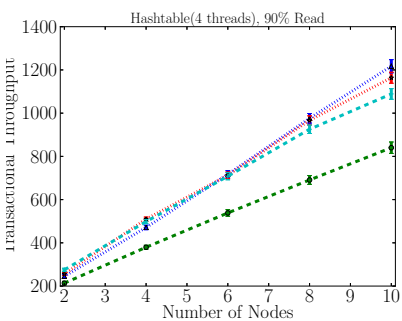

(e) HT (4 threads), 90\% Read

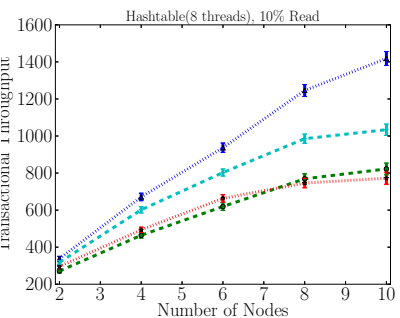

(c) HT (8 threads), 10\% Read

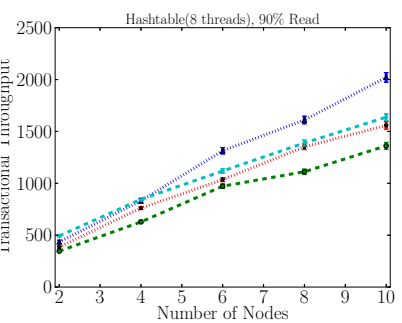

(f) HT (8 threads), 90\% Read

Fig. 4. Performance of DATS Using Hash Table (HT)

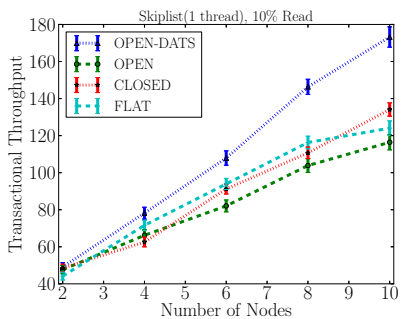

(a) SL (1 thread), 10\% Read

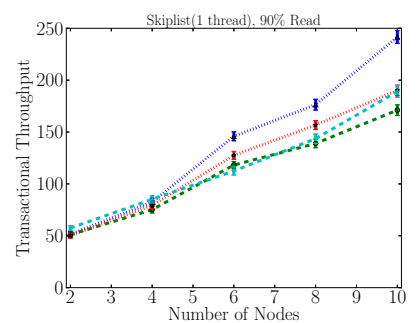

(d) SL (1 thread), 90\% Read

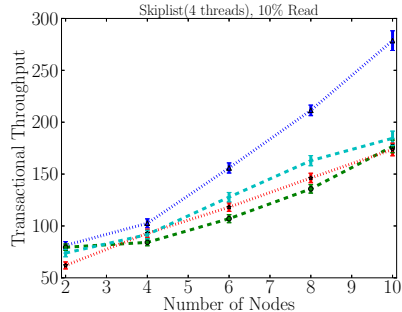

(b) SL (4 threads), 10\% Read

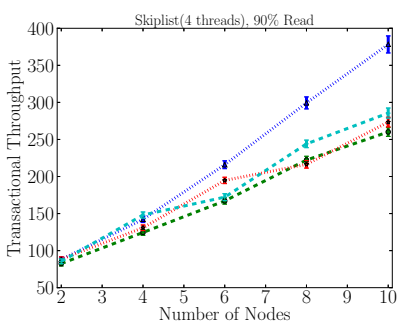

(e) SL (4 threads), 90\% Read

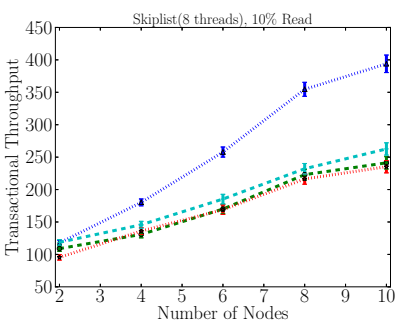

(c) SL (8 threads), 10\% Read

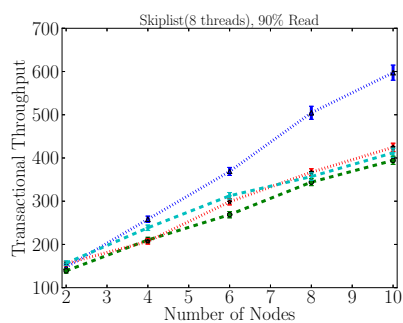

(f) SL (8 threads), 90\% Read

Fig. 5. Performance of DATS Using Skip List (SL) 


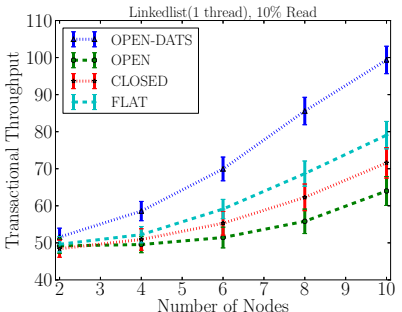

(a) LL (1 thread), 10\% Read

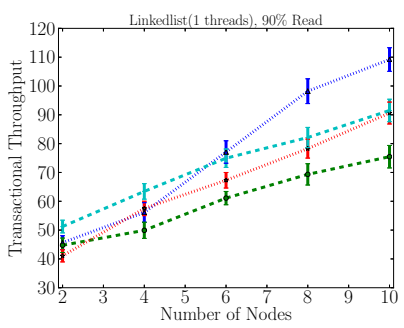

(d) LL (1 thread), 90\% Read

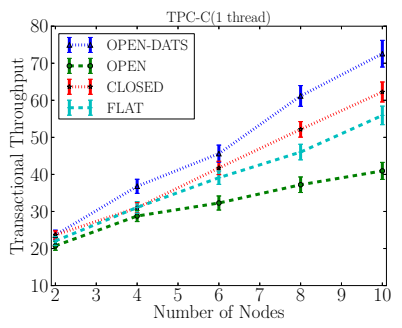

(g) TPC-C(1 thread)

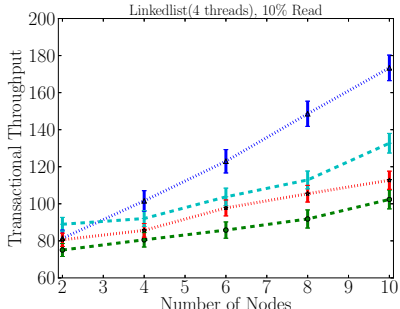

(b) LL (4 threads), 10\% Read

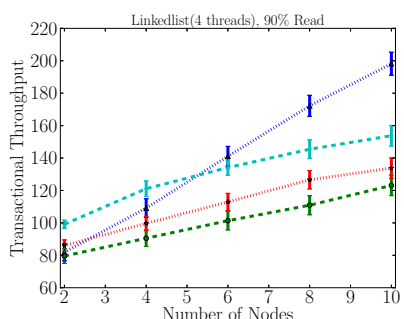

(e) LL (4 threads), 90\% Read

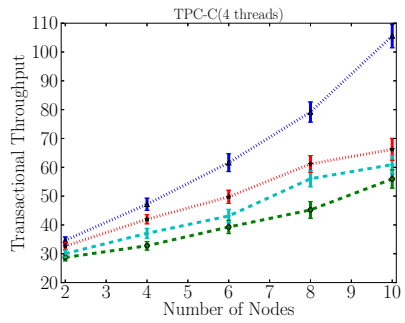

(h) TPC-C(4 threads)

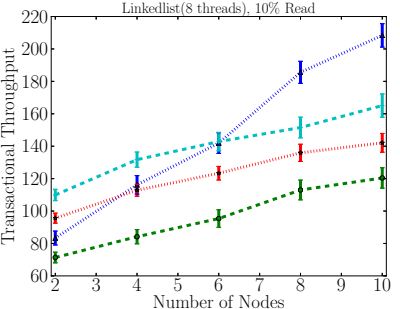

(c) LL (8 threads), 10\% Read

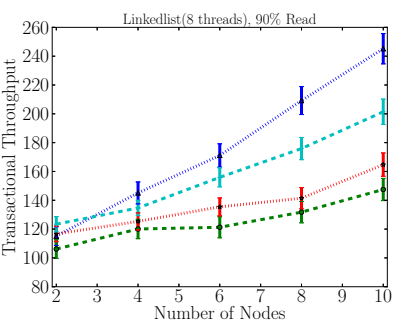

(f) LL (8 threads), 90\% Read

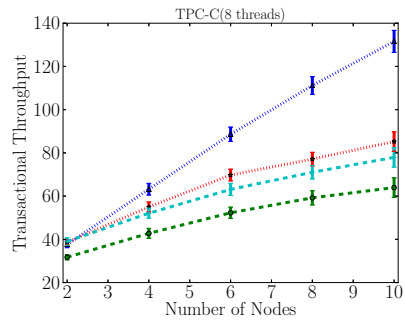

(i) $\mathrm{TPC}-\mathrm{C}(8$ threads $)$

Fig. 6. Performance of DATS Using Linked List (LL) and TPC-C

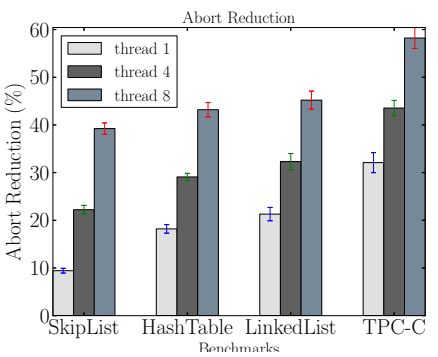

(a) \% Aborted transactions

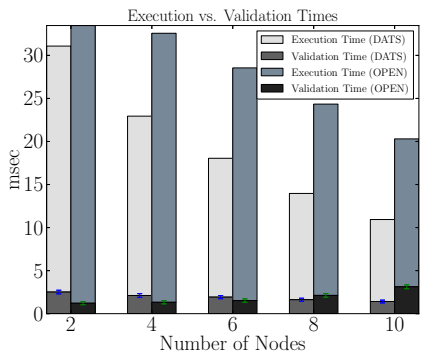

(b) Execution vs. Validation Times

Fig. 7. Analysis of Scheduling Overhead and Abort Reduction 
overheads of compensating actions and abstract locks. In 10\% read transactions, the number of aborts increases due to high contention. Outer transactions frequently abort, and corresponding compensating actions are executed; so DATS outperforms OPEN in throughput because it mitigates the abort of outer transactions and the corresponding compensating actions.

For the experiments with TPC-C in Figure 6(g) 6(h) 6(i) we used the amount of read and write transactions that its specification recommends. TPC-C benchmark accesses large tables to read and write values. Due to the non-negligible transaction execution time, the number of compensating actions and abstract locks in TPC-C significantly degrades the overall performance. Thus, DATS increases the performance in high contention (a large number of threads and nodes). By these results, it is evident how much unnecessary aborts of inner transactions affects performance and how much performance is improved through minimizing aborts. Even if DATS reduces the number of compensating actions and acquisition of abstract locks, the performance of OPEN is degraded because of the commit overheads of inner transactions [24; so the throughput of DATS is slightly better than CLOSED and FLAT, but significantly better than OPEN.

Figure 8 shows throughput speed-up relative to OPEN using Hash Table, Skip List, Linked List and TPC-C. Our results show that DATS performs up to $1.7 \times$ and $2.2 \times$ better than OPEN in micro-benchmarks and TPC-C, respectively.

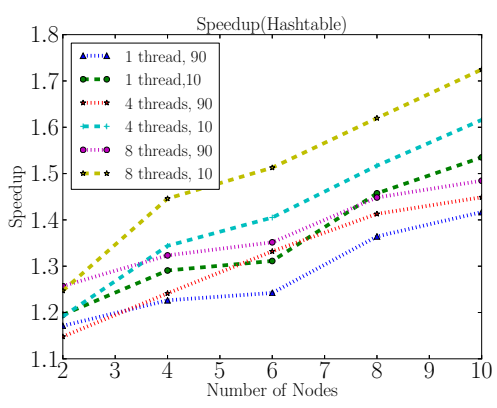

(a) Hash Table

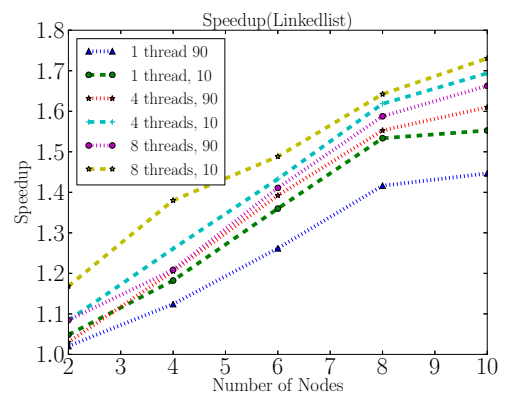

(c) Linked List

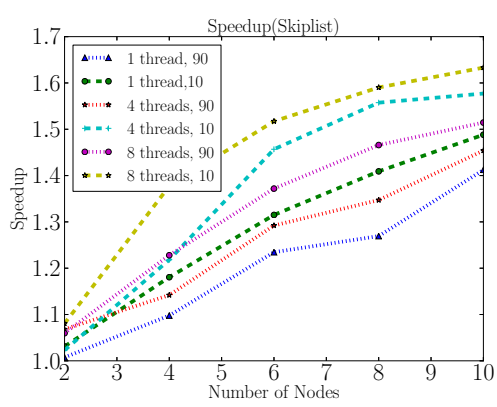

(b) Skip List

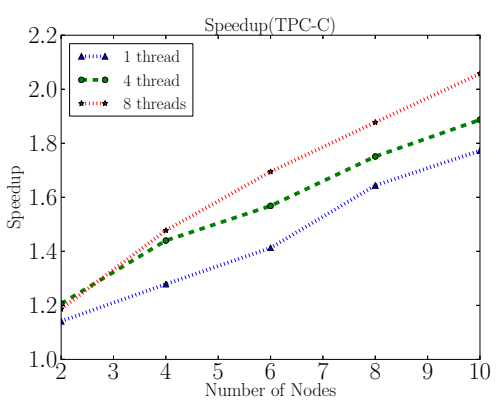

(d) TPC-C

Fig. 8. Speed-up (Throughput Relative to OPEN) in Hash Table, Skip List, Linked List, TPC-C 
Figure 7 shows the analysis of scheduling overhead and abort reduction. Checking dependencies occurs when a transaction validates, so we measure the average execution time and the average validation time of committed transactions as illustrated in Figure 7(b). The gap between the two validation times of DATS and OPEN proves the scheduling overhead. Even though the validation time of DATS is up to two times more than OPEN's, a large number of transactions validated simultaneously according to the increment of nodes, results in a shorten transaction response time, reducing the average validation time and aborts. Figure $7(\mathrm{a})$ the comparison between the percentage of aborted transactions of OPEN and DATS. As long as the number of threads increases, the number of aborts in DATS and OPEN increases too. However, the increasing abort ratio in DATS is less than in OPEN, proving how much DATS reduces the abort rate.

\section{Related Work}

Nested transactions (using closed nesting) originated in the database community and were thoroughly described in [18]. This work focused on the popular twophase locking protocol and extended it to support nesting.

Open nesting also originates in the database community [11, and was extensively analyzed in the context of undo-log transactions [26]. In these works, open nesting is used to decompose transactions into multiple levels of abstraction, and maintain serializability on a level-by-level basis.

One of the early works introducing nesting to Transactional Memory has been presented in [20]. They describe the semantics of transactional operations in terms of system states, which are tuples that group together a transaction ID, a memory location, a read/write flag, and the value read or written. They also provide sketches for several possible HTM implementations, which work by extending existing cache coherence protocols. They further focus on open nested transactions in [19, explaining how using multiple levels of abstractions can help in differentiating between fundamental and false conflicts and therefore improve concurrency. The authors of [17] implemented closed and open nesting in LogTM HTM. They implement nesting models by maintaining a stack of log frames, similar to the run-time activation stack, with one frame for each nesting level. In [1] the authors combined closed and open nesting by introducing the concept of transaction ownership. They propose the separation of TM systems into transactional modules (or Xmodules), which own data. Thus, a sub-transaction commits data owned by its own Xmodule directly to memory using an open-nested model. However, for data owned by foreign Xmodules, it employs the closed nesting model and does not directly write to the memory. The past closed nesting models 20171] have been studied for multiprocessor STM. N-TFA [25] and TFA-ON [24] are the first ever DTM implementation with support for closed and open-nesting, respectively, but do not consider transactional scheduling.

Transactional scheduling has been explored in a number of multiprocessor STM efforts [10/3 28|9|4]. In [10], the authors describe an approach that schedules transactions based on their predicted read/write access sets. In [3], they discuss the Steal-On-Abort transaction scheduler, which queues an aborted transaction 
behind the non-aborted transaction, and thereby prevents the two transactions from conflicting again. The Adaptive Transaction Scheduler (ATS) is present in [28], that adaptively controls the number of concurrent transactions based on the contention intensity: when the intensity is below a threshold, the transaction begins normally; otherwise, the transaction stalls and does not begin until dispatched by the scheduler. The CAR-STM scheduling approach is presented in [9], which uses per-core transaction queues and serializes conflicting transactions by aborting one and en-queuing it on another queue, preventing future conflicts. CAR-STM pre-assigns transactions with high collision probability (applicationdescribed) to the same core, thereby minimizing conflicts. In [5] they propose the Proactive Transactional Scheduler (PTS). Their scheme detects "hot spots" of contention that can degrade performance, and proactively schedules affected transactions around the hot spots. Attiya and Milani present the BIMODAL scheduler [4, which targets read-dominated and bimodal (i.e., those with only early-write and read-only) workloads. Kim and Ravindran extend the BIMODAL scheduler for DTM in [15]. Their scheduler, called Bi-interval, groups concurrent requests into read and write intervals, and exploits the tradeoff between object moving times (incurred in dataflow DTM) and concurrency of reading transactions, yielding high throughput. None of the past transactional schedulers for STM and DTM consider open-nested transactions.

\section{Conclusions}

When transactions with committed open-nested transactions conflict later and are re-issued, compensating actions for the open-nested transactions can reduce throughput. DATS avoids this by reducing unnecessary compensating actions, and minimizing inner transactions' remote abstract lock acquisitions through object dependency analysis. DATS shows the important of scheduling open-nested transactions in order to reduce the number of compensating actions and abstract locks in case of abort. Our implementation and experimental evaluation shows that DATS enhances transactional throughput for open-nested transactions over no DATS by as much as $1.7 \times$ and $2.2 \times$ with micro-benchmarks and real-application benchmark, respectively.

Acknowledgments. This work is supported in part by US National Science Foundation under grants CNS 0915895, CNS 1116190, CNS 1130180, and CNS 1217385 .

\section{References}

1. Agrawal, K., Lee, I.-T.A., Sukha, J.: Safe open-nested transactions through ownership. In: SPAA (2008)

2. Agrawal, K., Leiserson, C.E., Sukha, J.: Memory models for open-nested transactions. In: MSPC (2006)

3. Ansari, M., Luján, M., Kotselidis, C., Jarvis, K., Kirkham, C., Watson, I.: Steal-onabort: Improving transactional memory performance through dynamic transaction reordering. In: Seznec, A., Emer, J., O’Boyle, M., Martonosi, M., Ungerer, T. (eds.) HiPEAC 2009. LNCS, vol. 5409, pp. 4-18. Springer, Heidelberg (2009) 
4. Attiya, H., Milani, A.: Transactional scheduling for read-dominated workloads. In: Abdelzaher, T., Raynal, M., Santoro, N. (eds.) OPODIS 2009. LNCS, vol. 5923, pp. 3-17. Springer, Heidelberg (2009)

5. Blake, G., Dreslinski, R.G., Mudge, T.: Proactive transaction scheduling for contention management. In: Microarchitecture, pp. 156-167 (December 2009)

6. Couceiro, M., Romano, P., Carvalho, N., Rodrigues, L.: D2STM: Dependable distributed software transactional memory. In: PRDC (November 2009)

7. TPC Council. Tpc-c benchmark, revision 5.11 (February 2010)

8. Demmer, M.J., Herlihy, M.P.: The arrow distributed directory protocol. In: Kutten, S. (ed.) DISC 1998. LNCS, vol. 1499, pp. 119-133. Springer, Heidelberg (1998)

9. Dolev, S., Hendler, D., Suissa, A.: CAR-STM: scheduling-based collision avoidance and resolution for software transactional memory. In: PODC (2008)

10. Dragojević, A., Guerraoui, R., et al.: Preventing versus curing: avoiding conflicts in transactional memories. In: PODC 2009, pp. 7-16 (2009)

11. Garcia-Molina, H.: Using semantic knowledge for transaction processing in a distributed database. ACM Trans. Database Syst. 8(2), 186-213 (1983)

12. Herlihy, M., Koskinen, E.: Transactional boosting: a methodology for highlyconcurrent transactional objects. In: PPoPP 2008, pp. 207-216. ACM (2008)

13. Herlihy, M., Luchangco, V., Moir, M.: A flexible framework for implementing software transactional memory. In: OOPSLA, pp. 253-262 (2006)

14. Herlihy, M., Sun, Y.: Distributed transactional memory for metric-space networks. Distributed Computing 20(3), 195-208 (2007)

15. Kim, J., Ravindran, B.: On transactional scheduling in distributed transactional memory systems. In: Dolev, S., Cobb, J., Fischer, M., Yung, M. (eds.) SSS 2010. LNCS, vol. 6366, pp. 347-361. Springer, Heidelberg (2010)

16. Kim, J., Ravindran, B.: Scheduling closed-nested transactions in distributed transactional memory. IPDPS, 1-10 (2012)

17. Moravan, Bobba, Moore, Yen, Hill, Liblit, Swift, Wood: Supporting nested transactional memory in logTM. SIGPLAN Not. 41(11) (2006)

18. Moss, E.B.: Nested transactions: An approach to reliable distributed computing. Technical report, Cambridge, MA, USA (1981)

19. Moss, J.E.B.: Open-nested transactions: Semantics and support. In: Workshop of Memory Performance Issues (2006)

20. Moss, J.E.B., Hosking, A.L.: Nested transactional memory: model and architecture sketches. Sci. Comput. Program. 63, 186-201 (2006)

21. Palmieri, R., Quaglia, F., Romano, P.: Osare: Opportunistic speculation in actively replicated transactional systems. In: SRDS (2011)

22. Saad, M., Binoy, R.: Supporting STM in distributed systems: Mechanisms and a Java framework. In: ACM SIGPLAN Workshop on Transactional Computing 2011 (2011)

23. Saad, M.M., Ravindran, B.: HyFlow: a high performance distributed software transactional memory framework. In: HPDC 2011 (2011)

24. Turcu, R.: On open nesting in distributed transactional memory. In: SYSTOR (2012)

25. Turcu, A., Ravindran, B.: On closed nesting in distributed transactional memory. In: Seventh ACM SIGPLAN workshop on Transactional Computing (2012)

26. Weikum, G.: Principles and realization strategies of multilevel transaction management. ACM Trans. Database Syst. 16(1), 132-180 (1991)

27. Yang, Menon, Ali-Reza, Antony, Hudson, Moss, Saha, Shpeisman: Open nesting in software transactional memory. In: PPoPP. ACM, New York (2007)

28. Yoo, R.M., Lee, H.-H.S.: Adaptive transaction scheduling for transactional memory systems. In: SPAA, pp. 169-178 (2008) 